\title{
Search for new physics with long-lived particles in ATLAS
}

\section{Antonio POLICICCHIO*}

University of Washington, Seattle (WA), USA

E-mail: antonio.policicchiodcern.ch

On behalf of the ATLAS Collaboration

Various models of new physics, including Hidden Valley models and some supersymmetric models, predict the existence of long-lived particles decaying a significant distance away from the interaction point or traveling inside the detector with $\beta<1$. These characteristics make their detection difficult using standard LHC-detector triggers. This paper briefly presents the prospects for searches, trigger and detection in the ATLAS detector [1] of these long-lived particles.

European Physical Society Europhysics Conference on High Energy Physics, EPS-HEP 2009,

July 16 - 222009

Krakow, Poland

${ }^{*}$ Speaker. 


\section{Long-lived charged particles in ATLAS}

Long-lived charged particles have a common characteristic in many models. While the velocity $(\beta)$ spectrum of these particles is model dependent, the shared characteristic is a heavy long-lived charged particle with $\beta$ significantly smaller than 1 . This unique signature makes it possible to focus on model independent searches. Reference [2] outlines a strategy for the detection of exotic massive, long-lived hadrons (so-called R-hadrons) formed from either stable gluinos or stops considered in the context of a Split-SUSY and NLSP scenarios. Table 1 shows the acceptance numbers and rates for the various R-Hadron masses. It can be seen that for R-hadron masses below $1 \mathrm{TeV}$ ATLAS opens up a discovery window with integrated luminosity of the order of $1 \mathrm{fb}^{-1}$ at $14 \mathrm{TeV}$ center of mass energy. For masses above $1 \mathrm{TeV}$ the rate of signal events is small, and is comparable to the expected background rate, so discovery would be challenging.

The work in [2] does not fully exploit the capabilities of the ATLAS detector: for instance, timing information was not used. Because the stable charged particles can be slow, the detector hits may occur in the bunch crossing (BC) following the event BC, which can compromise the detection efficiency. New techniques have been developed in order to recover the detector hits from the next $\mathrm{BC}$ avoiding a deterioration of the trigger efficiency. These techniques allow identification of a heavy charged particle by estimating the particle velocity and mass from the hit time in the trigger chambers and by selecting $\beta$ that minimizes the $\chi^{2}$ of the

\begin{tabular}{c|c} 
Sample & Rate $($ Events/fb \\
& -1 \\
\hline $300 \mathrm{GeV}$ gluino & $6.44 \times 10^{3}$ \\
$600 \mathrm{GeV}$ gluino & $2.70 \times 10^{2}$ \\
$1000 \mathrm{GeV}$ gluino & 10.7 \\
$1600 \mathrm{GeV}$ gluino & 0.147 \\
\hline $300 \mathrm{GeV}$ stop & 70.0 \\
$600 \mathrm{GeV}$ stop & 3.9 \\
$1000 \mathrm{GeV}$ stop & 0.1 \\
\hline $\mathrm{QCD}$ & 0.9 \\
electroweak $(Z \rightarrow \mu \mu)$ & 0.8
\end{tabular}

Table 1: Number of events selected for the various R-Hadron masses. Results have been obtained for $p \bar{p}$ collisions at $\sqrt{s}=14 \mathrm{TeV}$. track. Details can be found in [3]. These were tested in the framework of a GMSB model that includes sleptons with a mass of $100 \mathrm{GeV}$, and $300 \mathrm{GeV}$ R-Hadrons produced in a split SUSY model. For these two models, given well calibrated data, discovery is possible at very low luminosity. We note that the techniques presented in [3] require only the presence of a slow particle, and thus the results are model independent.

\section{Trigger for long-lived neutral particles in ATLAS}

For the purpose of exploring the challenges to the trigger posed by long-lived neutral particles, the Hidden Valley (HV) [4] scenario serves as an excellent setting. In the HV models long-lived particles that decay to heavy quark pairs can be produced in SUSY processes, $Z^{\prime}$ decays and Higgs boson decays. Results are presented of a study of a reference process of the ATLAS Detector performance for the Higgs decay $h^{0} \rightarrow \pi_{v}^{0} \pi_{v}^{0}$, where $\pi_{v}^{0}$ is neutral and has a displaced decay to bottom quarks [5]. The standard ATLAS triggers [2] are able to select only a small fraction of these unique events. However, it is possible to use the displaced decay signature as a trigger object in order to increase the fraction of events accepted. We consider three detector regions and illustrate signature 


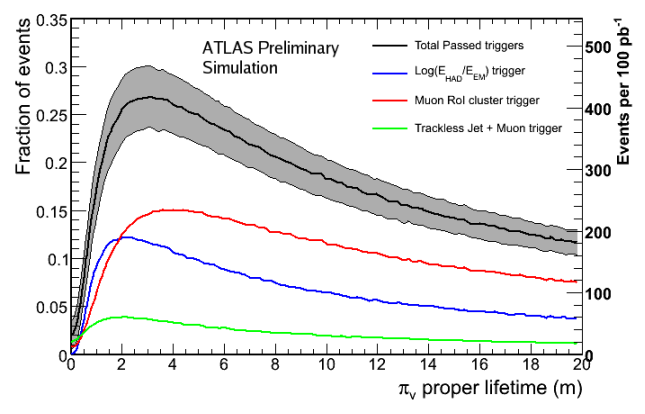

Figure 1: $h^{0} \rightarrow \pi_{v}^{0} \pi_{v}^{0}$ events accepted by our long-lived particle triggers vs the $\pi_{v}^{0}$ lifetime. Left axis: fraction of events accepted. Right axis: number of events for an integrated luminosity of $100 \mathrm{pb}^{-1}$ assuming $\operatorname{Br}\left(h^{0} \rightarrow\right.$ $\left.\pi_{v}^{0} \pi_{v}^{0}\right)=100 \%$. A $140 \mathrm{GeV}$ mass for the $h^{0}$ and $40 \mathrm{GeV}$ for the $\pi_{v}^{0}$ have been assumed, at $10 \mathrm{TeV}$ cm energy.

driven triggers that can be used to preferentially select such Hidden Valley events:

1) Decays in the Muon Spectrometer (MS) produce a large number of charged hadrons in a small $(\eta ; \phi)$ region, resulting in a large number of clustered muon first level trigger objects (Regions of Interest (RoI)) accompanied by a lack of activity in the Inner Detector (ID) and Calorimeters. These events are rejected by the current ATLAS High Level Trigger (HLT) trigger which requires a matching ID track for the first level trigger muons. However, the cluster of RoIs can be used as HLT trigger to select these HV events decaying in the MS with an efficiency of $\sim 70 \%(\sim 25 \%)$ in the barrel (endcap) and with minimal SM backgrounds.

2) Decays occuring inside the Hadronic Calorimeter (HCal) are characterized by jets with few/no tracks in the ID and little energy deposited in the EM Calorimeter (ECal). This leads to jets with more energy deposited in the HCal than in the ECal. The logarithm of the hadronic to electromagnetic energy ratio for these decays changes from a characteristic negative for Standard Model jets to a positive value with no expected activity in the ID. This jet characteristic has been used to build a HLT object selecting decays in the barrel (endcap) HCal with an efficiency of $\sim 60 \%(\sim 30 \%)$.

3) Decays in the outer ID lead to jets with no connecting track to the IP (trackless jets). The QCD background can be significantly reduced by requiring that the trackless jet contain at least a muon from semileptonic decays of the $b$ quark in the jet cone. A trackless jet with a muon in the jet cone is the trigger object $(\sim 2 \%$ efficient in the outer ID).

4) Decays in the Beam Pipe or in the innermost ID should be found by the $b$-tagging algorithms with good efficiency and new triggers are not needed.

We have implemented in the ATLAS simulation package the three new signature-based triggers [5]. Figure 1 shows the expected fraction of detected events vs the $\pi_{v}^{0}$ lifetime. We expect a significant fraction of events in a wide range of lifetimes. Applying these trigger objects on simulated di-jet samples results in a negligible trigger rate. Nevertheless, trigger background from Standard Model QCD processes will need to be better understood with real data.

\section{References}

[1] G. Aad et al., The ATLAS Collaboration, JINST 3 S08003.

[2] G. Aad et al., The ATLAS Collaboration, CERN-OPEN-2008-020.

[3] S. Tarem et al., EPJ C62 Issue 2 (2009) 281.

[4] M. J. Strassler and K. M. Zurek, Phys. Lett. B 651 (2007) 374-379.

[5] G. Aad et al., The ATLAS Collaboration, ATL-PHYS-PUB-2009-082. 